\title{
Dielectronic satellite spectra of heliumlike iron and nickel from the unified recombination method
}

\author{
Sultana N. Nahar* and Anil K. Pradhan ${ }^{\dagger}$ \\ Department of Astronomy, The Ohio State University, Columbus, Ohio 43210, USA
}

(Received 10 March 2006; published 22 June 2006)

\begin{abstract}
It is shown that resonances in the unified recombination cross sections correspond directly to dielectronic satellite spectra. Positions, intensities, and profiles of satellite lines may be obtained accurately using the Breit-Pauli $R$-matrix method and the unified theory of total electron-ion recombination. The intrinsic dielectronic resonance strength corresponding to autoionizing resonances is defined in analogy with measured quantities, and with resonance oscillator strengths. Satellite intensities then depend extrinsically on dynamical parameters in the plasma and may be obtained at any temperature in a straightforward manner from the resonance strengths. Profiles of dielectronic satellite lines of He-like Fe xxv and Ni xxvII are obtained in an $a b$ initio manner. Detailed calculations also exhibit interference effects in resonance profiles delineated at high resolution. Although interference among resonances is not important for resonance strengths of the highly charged ions $\mathrm{Fe}$ XXV and Ni XXVII it might be significant for lower ionization states of heavy elements in general. The resonance identitification scheme for unified recombination cross sections is described, and applied to obtain all known $K L L$ satellites of Fe xxv and Ni xxvII. Dielectronic satellite strengths are compared with earlier works and significant differences and similarities are discussed.
\end{abstract}

DOI: 10.1103/PhysRevA.73.062718

PACS number(s): 32.80.Fb, 32.30.Rj, 32.70.Fw, 32.80.Cy

\section{INTRODUCTION}

The dielectronic recombination process manifests itself through the radiative decay of an autoionizing state of the $(e$, ion) system. The resonances are formed from doubly excited states following electron impact excitation of a dipole core transition and capture of the incident electron. These resonances appear as "satellite" lines at energies somewhat lower than the energy of the principal dipole core transition $[1,2]$, and are otherwise indistinguishable from ordinary emission lines. X-ray spectra of highly charged ions exhibit dielectronic satellites which, in terms of wavelengths, are at longer wavelengths (or "redward") than the principal line of the $1 s^{2}{ }^{1} S_{0}-1 s 2 p^{1} P_{1}^{o}$ transition (we eschew the phrase "resonance line" common in the literature to avoid obvious confusion). In particular, H-like and He-like ions of high- $Z$ elements have strong satellite lines owing to large radiative decay rates of core transitions which compete effectively with typical autoionization rates $A_{a} \sim 10^{13}-10^{14} \mathrm{~s}^{-1}$.

The dielectronic satellites are sensitive plasma diagnostics of electron temperature since they are excited only by electrons at precise resonance energies, in contrast to boundbound core transitions excited by all electrons with energies higher than the upper level. As such the ratio of the satellite line intensity to the principal line is a useful indicator of electron temperature. Its utility is further enhanced by the fact that the satellite intensity depends only on the ionization state of a given ion although the radiative decay involves the ( $e$, ion) system, i.e., independent of the ionization balance in the plasma. Owing to their extreme temperature sensitivity the strengths of dielectronic satellites observed from a source may indicate heating of ionizing plasma on the one hand, and

\footnotetext{
*Electronic address: nahar@astronomy.ohio-state.edu

†Electronic address: pradhan@astronomy.ohio-state.edu
}

cooling of recombining plasma on the other hand.

The spectra of He-like ions are of particular diagnostic value in $\mathrm{X}$-ray spectroscopy of high-temperature sources above $10^{6} \mathrm{~K}$. For $T \sim 10^{7} \mathrm{~K}$ or higher, corresponding to the $\mathrm{keV}$ range in energy, He-like $\mathrm{Fe}$ and $\mathrm{Ni}$ may exist in the source [3] with strong dielectronic satellite spectra under appropriate conditions. Toward the lower-temperature range dielectronic satellites are prominently seen in x-ray spectra of Fe XXV and Ni XXVII. At temperatures significantly below that of maximum abundance in coronal equilibrium the dielectronic satellites dominate the spectra of the $K \alpha$ complex of Fe xxv. Detailed modeling of stationary and transient plasmas shows that owing to the dielectronic satellites the total $K \alpha$ intensity is shifted toward lower energies up to $100 \mathrm{eV}$ below the principal transition $1{ }^{1} S_{0}-2{ }^{1} P_{1}^{o}$ at $6.7 \mathrm{keV}$ (the $w$ line), whereas at high temperatures the dielectronic satellites vanish and the intensity is primarily in the $w$ line $[4,5]$. This has important consequences in ascertaining the nature of the plasma in the source. Iron in much lower ionization stages, less ionized than Ne-like with a filled $2 p$ shell, gives rise to the well-known fluorescent emission $K \alpha$ line at $6.4 \mathrm{keV}$ formed in low-temperature sources such as the innermost stable region in accretion disks around black holes. It has been pointed out $[4,5]$ that in higher-temperature regions in active galactic nuclei that are thought to harbor supermassive black holes, such as Sagittarius $A^{*}$ at the galactic center of the Milky Way [6], x rays are emitted from the He-like $6.7 \mathrm{keV} K \alpha$ complex, as well as at energies between 6.6 and $6.7 \mathrm{keV}$ due to the dielectronic satellites of Fe xxv. Therefore the precise observed energy of the $K \alpha$ complex, dependent on the dielectronic satellites, is potentially a discriminant of the temperature, dynamics, composition, and other extrinsic macroscopic variables in a variety of sources and regions therein.

In previous works (e.g., [7-9]) we have described a unified method for $(e$, ion) recombination that subsumes both 
the resonant dielectronic recombination (DR) process and the background nonresonant radiative recombination (RR) process in an $a b$ initio manner in the close-coupling (CC) $R$-matrix method. The resulting CC calculations may employ eigenfunction expansions for the core ion that enable selfconsistent computations of photoionization and electron impact excitation processes as well. The unified method also has the advantage of general applicability to all atomic systems, from neutral to highly ionized, as in close-coupling calculations for photoionization and electron impact excitation. A large number of such calculations have been carried out for many atomic systems (Ref. [9] and references therein). However, we have not heretofore explored the utility of the method for the dielectronic satellite spectra and computation of satellite line intensities, although the resonances in the unified $(e$, ion) recombination cross sections correspond, in principle, directly to the observed satellite lines [10]. Radiation damping of resonances in unified cross sections has been described in a previous work [11] to obtain autoionization and radiative decay rates of resonances of the (e, Fe xxv) system, and compared with other methods which employ the individual resonance approximation (IRA). In this work, we generalize the approach to obtain the dielectronic satellite strengths directly from unified recombination cross sections $\left(\sigma_{R C}\right)$. This also establishes an analogy with the familiar oscillator strength to quantify the resonance strength as an intrinsic quantity; in previous works we have obtained accurate photoabsorption resonance oscillator strengths from high-precision photoionization cross sections (e.g., $[12,13])$. The resonance profiles for the dielectronic satellites are in an ab initio manner, not by previous methods. The profiles exhibit interference effects related to configuration interaction among competing channels that can affect dielectronic satellite intensities. We show that the dielectronic satellite intensity may thereby be obtained in combination with extrinsic quantities depending only on the electron temperature. The calculations are carried out for two important He-like ions in laboratory and astrophysical sources, Fe xxv and Ni xxviI.

The present unified results are compared with other approaches based on the independent resonance approximation for the computation of autoionization and radiative rates separately. There have been several such calculations [14-17] in the IRA, but otherwise using different theoretical methods to compute the atomic structure and the continuum wave functions. Experimental measurements have been carried out for Fe xxv on the electron beam ion trap (EBIT) at Lawrence Livermore National Laboratory [18]. A comparison of the experimental results with previous theoretical calculations showed generally good agreement, but with dispersions for individual dielectronic satellites of up to about $30 \%$. A prime motivation for the present work is to compare and contrast our unified method with previous methods based on the IRA. It is also of interest to compare with experimental measurements which, like the unified cross sections, obtain total resonant and nonresonant contributions that are naturally indistinguishable within the limited range of energy associated with the finite width of the dielectronic satellites. Finally, from the practical point of view it is important to ascertain whether any differences are found with available data that might be of significance in laboratory and astrophysical applications, especially for highly charged ions where we expect general agreement among all methods.

\section{THEORY}

The most prominent satellite lines correspond to the $K L L$ resonances $(1 s 2 \ell 2 \ell)$ in the recombination spectrum of a three-electron recombined ion. There are 22 satellite lines that form from the $n=2$ autoionizing levels of configurations $1 s 2 s^{2}, 1 s 2 p 2 s, 1 s 2 p^{2}$. The profiles and intensities of these lines can be obtained in an ab initio manner from the unified method of recombination. All KLL resonances can be isolated and identified in the unified recombination cross sections of the three final recombined levels (i) $1 s^{2} 2 s\left({ }^{2} S_{1 / 2}\right.$ ), (ii) $1 s^{2} 2 p\left({ }^{2} P_{1 / 2}^{o}\right)$, and (iii) $1 s^{2} 2 p\left({ }^{2} P_{3 / 2}^{o}\right)$ as will be demonstrated.

Photorecombination of an incident electron with the target ion may occur through (i) the nonresonant background continuum-radiative recombination,

$$
e+X^{2+} \leftrightarrow h \nu+X^{+},
$$

which is the inverse process of direct photoionization, or (ii) a two-step recombination process via autoionizing resonances, i.e., dielectronic recombination,

$$
e+X^{2+} \leftrightarrow\left(X^{+}\right)^{* *} \leftrightarrow\left\{\begin{array}{c}
\text { (i) } e+X^{2+}, \\
\text { (ii) } h \nu+X^{+} .
\end{array}\right.
$$

The intermediate quasibound doubly excited autoionizing state leads either to (i) autoionization, a radiationless transition to a lower target state with the electron going into a continuum, or (ii) radiative stabilization to a recombined bound state via decay of the ion core (usually to the ground state) with the electron captured. It is the latter process that manifests itself as the dielectronic satellites corresponding, in principle, to the infinite series of autoionizing levels converging on to the threshold for the principal core transition. However, in practice, only the lowest $n$ complexes are observed and resolved; the higher ones generally blend with the principal line. For He-like ions we consider only the dielectronic satellites corresponding to autoionizing levels $1 \mathrm{~s} 2 \ell, 2 \ell^{\prime}$ of the $K L L$ complex.

The unified method [7-9] subsumes both the RR and DR processes. It considers photoionization from and recombination into the infinity of levels of the $(e+$ ion $)$ system. The recombined levels are divided into two groups of bound levels: group A levels with $n \leq n_{o}$ and all possible fine structure $J \pi$ symmetries, and group B levels with $n_{o}<n \leq \infty$; where $n_{o}$ is typically 10 . Detailed photoionization cross sections $\sigma_{\mathrm{PI}}$ including autoionizing resonances are obtained for a large number of bound states with $n \leq n_{\mathrm{o}} \sim 10$ and are treated with principle of detailed balance for the recombination cross sections $\sigma_{R C}$. Calculations are carried out in the close-coupling approximation.

In the $\mathrm{CC}$ approximation the target ion (core) is represented by an $N$-electron system while the $(N+1)$ th electron is the interacting electron. The total wave function $\Psi(E)$ of the $(N+1)$ electron-ion system of symmetry $J \pi$ is represented in terms of an expansion of target eigenfunctions as 


$$
\Psi(\text { ion }+e ; E)=A \sum_{i} \chi_{i}(\text { ion }) \theta_{i}+\sum_{j} c_{j} \Phi_{j}(\text { ion }+e) .
$$

$\chi_{i}$ is the target wave function in a specific level $J_{i} \pi_{i} . \theta_{i}$ is the wave function for the $(N+1)$ th electron in a channel labeled as $S_{i} L_{i}\left(J_{i}\right) \pi_{i} k_{i}^{2} \ell_{i}(J \pi)$ where $k_{i}^{2}$ is its incident kinetic energy. $A$ is the antisymmetrization operator. The $\Phi_{j}$ 's are functions of the $(N+1)$-electron system that account for short-range correlation and the orthogonality between the continuum and the bound orbitals.

In the relativistic Breit-Pauli $R$-matrix (BPRM) method, developed under the Iron Project [19], the set of $S L \pi$ are recoupled for $J \pi$ levels of the $(e+$ ion) system, followed by diagonalization of the Breit-Pauli Hamiltonian $H_{N+1}^{\mathrm{BP}} \Psi=E \Psi$, where

$$
H_{N+1}^{\mathrm{BP}}=H_{N+1}^{\mathrm{NR}}+H_{N+1}^{\mathrm{mass}}+H_{N+1}^{\mathrm{Dar}}+H_{N+1}^{\mathrm{so}} .
$$

The first term $H_{N+1}^{\mathrm{NR}}$ is the nonrelativistic Hamiltonian

$$
H_{N+1}^{\mathrm{NR}}=\sum_{i=1}^{N+1}\left(-\nabla_{i}^{2}-\frac{2 Z}{r_{i}}+\sum_{j>i}^{N+1} \frac{2}{r_{i j}}\right),
$$

and the additional one-body terms are

$$
\begin{gathered}
H^{\mathrm{mass}}=-\frac{\alpha^{2}}{4} \sum_{i} p_{i}^{4} \quad(\text { mass correction term }), \\
H^{\mathrm{Dar}}=\frac{Z \alpha^{2}}{4} \sum_{i} \nabla^{2}\left(\frac{1}{r_{i}}\right) \quad(\text { Darwin term }), \\
\left.H^{\mathrm{so}}=Z \alpha^{2} \sum_{i} \frac{1}{r_{i}^{3}} \ell_{i} \cdot \mathbf{s}_{i} \quad \text { (spin-orbit interaction term }\right),
\end{gathered}
$$

respectively. The spin-orbit term splits the $L S$ term into fine structure components. The positive $\left(E=k^{2}>0\right)$ and negative $\left(E=k^{2}<0\right)$ energy states of Eq. (4) define the continuum $\Psi_{F}$ or bound $\Psi_{B}$ states of the (e+ion) system. Coupling of closed and open channels in the transition matrix introduces the autoionizing resonances.

As mentioned above, photoionization and recombination calculations include autoionizing resonances for all group A levels. The reduced transition matrix element for the boundfree photoionization or recombination processes is given by

$$
\left\langle\Psi_{B}\|\mathbf{D}\| \Psi_{F}\right\rangle,
$$

where $\mathbf{D}$ is the dipole operator. $\mathbf{D}=\Sigma_{i} r_{i}$, in length form where the sum is over the number of electrons. The photoionization cross section is obtained as

$$
\sigma_{\mathrm{PI}}=\frac{1}{g_{i}} \frac{4 \pi^{2}}{3 c} \omega \mathbf{S},
$$

where $g_{i}$ is the statistical weight factor of the initial bound state and $\mathbf{S}=\left|\left\langle\Psi_{B}\|\mathbf{D}\| \Psi_{F}\right\rangle\right|^{2}$ is the dipole line strength. For highly charged $\mathrm{H}^{-}$and He-like recombining ions, the probability of radiative decay of an autoionizing state is often comparable to that of autoionization (typically $10^{12}-10^{14} \mathrm{~s}^{-1}$, discussed in Ref. [20]). With strong dipoleallowed $2 p\left({ }^{2} P_{1 / 2,3 / 2}^{o}\right) \rightarrow 1 s\left({ }^{2} S_{1 / 2}\right)$ transitions in $\mathrm{H}$-like and
$1 s 2 p\left({ }^{1} P_{1}^{o}\right) \rightarrow 1 s^{2}\left({ }^{1} S_{0}\right)$ transitions in He-like ions, autoionizing resonances are radiatively damped to a significant extent. The radiative damping effect of all near-threshold resonances, up to effective quantum number $\nu \leq 10$, can be considered using the resonance fitting procedure as described in $[11,21,22]$.

The photorecombination cross section $\sigma_{\mathrm{RC}}$ is related to the photoionization cross section $\sigma_{\mathrm{PI}}$ through the principle of detailed balance (Milne relation) as

$$
\sigma_{\mathrm{RC}}(\epsilon)=\frac{\alpha^{2}}{4} \frac{g_{i}}{g_{j}} \frac{(\epsilon+I)^{2}}{\epsilon} \sigma_{\mathrm{PI}}
$$

in Rydberg units; $\alpha$ is the fine-structure constant, $\epsilon$ is the photoelectron energy, $g_{j}$ is the statistical weight factor of the recombined ion, and $I$ is the ionization potential. $\sigma_{\mathrm{RC}}$ are computed from the photoionization cross sections at a sufficiently large number of energies to delineate the nonresonant background and the autoionizing resonances, thereby representing both radiative and dielectronic recombination processes. It is assumed that the recombining ion is in the ground state, and recombination can take place into the ground or any of the excited recombined $(e+$ ion) states.

The resonances in unified recombination cross sections $\sigma_{\mathrm{RC}}$ at the satellite energies are the dielectronic satellite lines. The lines in the $K \alpha$ complex form from and lie well within the energy ranges of the group A states. Hence, treatment of the resonances beyond $\nu=10$ is not needed. However, the background contributions of group B states do contribute to the total spectrum and are included in the present calculations as explained by Nahar [23]. Integration or averaging over the resonance profiles in a numerically precise manner provides (a) the recombination resonance strengths which would be intrinsic quantities independent of external plasma conditions, and (b) the dielectronic satellite intensities which would depend on the electron temperature. The satellite line intensities are computed in an analogous manner to unified recombination rate coefficients $\alpha_{R}(T)$ defined as

$$
\alpha_{R}(T)=\frac{4}{\sqrt{2 \pi m}}\left(\frac{1}{k T}\right)^{3 / 2} \int_{0}^{\infty} \epsilon e^{-\varepsilon / k T} \sigma_{\mathrm{RC}} d \epsilon .
$$

However, for the narrow dielectronic satellites, where the exponential factor varies little over the resonance energy range $\epsilon_{i}-\epsilon_{f}$, the satellite rate coefficient can be written as

$$
\alpha_{s}(T)=\frac{4}{\sqrt{2 \pi m}} \frac{e^{-\epsilon_{s} / k T}}{(k T)^{3 / 2}} \int_{\epsilon_{i}}^{\epsilon_{f}} \epsilon \sigma_{\mathrm{RC}} d \epsilon
$$

where $\epsilon_{s}$ is the central energy of the resonance. We define the temperature-independent dielectronic resonance strength for the satellite $s$ as

$$
S_{\mathrm{RC}}(s)=\int_{\epsilon_{i}}^{\epsilon_{f}} \epsilon \sigma_{\mathrm{RC}} d \epsilon
$$

Multiplying $S_{\mathrm{RC}}(s)$ by the temperature-dependent factor 


$$
f(T)=\frac{4}{\sqrt{2 \pi m}} \frac{e^{-\epsilon_{s} / k T}}{(k T)^{3 / 2}}=0.015484 \frac{e^{-\epsilon_{s} / k T}}{T^{3 / 2}},
$$

the recombination rate coefficient of the dielectronic satellite $s$,

$$
\alpha_{s}(T)=S_{\mathrm{RC}} f(T),
$$

can be obtained at any temperature $T$. We use the units Ry for $\epsilon, \mathrm{Mb}$ for $\sigma_{\mathrm{RC}}$, and $\mathrm{K}$ for $T$. The units of $S_{\mathrm{RC}}$ can be converted to cgs units by using $\mathrm{Ry}^{2} \mathrm{Mb}=4.75109$ $\times 10^{-40} \mathrm{erg}^{2} \mathrm{~cm}^{2}$ where $1 \mathrm{Ry}=2.1797 \times 10^{-11} \mathrm{ergs}$ and $1 \mathrm{Mb}=10^{-18} \mathrm{~cm}^{2}$. This conversion factor for $S_{\mathrm{RC}}$ together with $m=9.109 \times 10^{-28} \mathrm{~g}$ and $k=1.380658 \times 10^{-16} \mathrm{ergs} / \mathrm{K}$ gives the numerical factor 0.015484 in $f(T)$.

The unified method enables direct computation of the quantity $S$ defined in terms of the energy-dependent cross section for dielectronic recombination, $\sigma_{s}$, measured in the EBIT experiment [18],

$$
S(s)=\int_{\epsilon_{i}}^{\epsilon_{f}} \sigma_{\mathrm{RC}}(s) d \epsilon .
$$

Because of the narrow energy widths of the dielectronic satellites, $S_{s}$ can further be approximated from $S_{\mathrm{RC}}(s)$ as

$$
S(s)=S_{\mathrm{RC}}(s) / \epsilon_{s} .
$$

In order to obtain $S(s)$ in units of $\mathrm{eV} \mathrm{cm}{ }^{2}$, as commonly used in experiments, the right hand side should be multiplied by $13.6057 \times 10^{-18}$.

At this point we note the analogous expression for resonance absorption oscillator strengths

$$
f_{r}=\int_{r}\left(\frac{d f}{d \epsilon}\right) d \epsilon=\int_{r} \sigma_{\mathrm{PI}} d \epsilon
$$

as obtained in our previous work (e.g., in Ref. [13]) from photoionization cross sections.

In the unified method $\sigma_{\mathrm{RC}}$ is obtained as the summed contributions from level-specific $\sigma_{\mathrm{PI}}$ of all final recombined states and angular momenta using the detailed balance. The method thus differs from previous approximations based on the IRA in fundamental ways. The IRA methods follow the prescription first laid down in Refs. [1,2], wherein the satellite intensities are computed from electron capture rate coefficients obtained in terms of the autoionization and radiative decay rates computed independently at a single resonance energy $E_{s}$. For example, in Ref. [15] the intensity of the satellite is obtained as

$$
I_{s}=2.06 \times 10^{-16} \frac{e^{E_{s} / k T}}{T^{3 / 2}}\left(\frac{g_{s} A_{a}(s) A_{r}(s)}{\sum A_{a}(s)+\sum A_{r}(s)}\right)
$$

where the expression in large parentheses involves the branching ratio between autoionization and radiative channels. $A_{a}$ is the total autoionization rate related to the innershell excitation rate by detailed balance, and $A_{r}$ is the radiative decay rate for the specific satellite transition $s . A_{a}$ is computed by summing over all final channels and angular momenta. On the other hand, the unified method employs photoionization cross sections for all recombined (e,ion) states up to a given $n_{o}(S L J \pi)$ (usually $\left.n_{o} \leq 10\right)$, and DR theory [8,24] for $n_{o}<n \leq \infty$. Satellite resonances correspond to low angular momenta and appear in the detailed recombination cross sections. But in principle the unified method includes the summation over all continua, $\epsilon J \pi$, that constitute the total autoionization rate $A_{a}$. In practice, $J$ is usually in the range 10-12 for both even and odd parities of the electron-ion system.

Finally, in observations or experimental measurements the $K L L$ dielectronic satellites due to recombination are usually seen together with the resonance line $w$ due to electron impact excitation of the transition $1{ }^{1} S_{0}-2{ }^{1} P_{1}^{o}$ in He-like ions. Therefore we can obtain the intensity ratio for practical applications as

$$
\frac{I_{s}}{I_{w}}=\frac{\alpha_{s}}{q_{w}}
$$

where $\alpha_{s}$ is the satellite rate coefficient and $q_{w}$ is the excitation rate coefficient of the $w$ line.

\section{COMPUTATIONS}

We consider photoionization and recombination as inverse processes:

$$
\begin{aligned}
& h \nu+\mathrm{Fe} \text { xxIv } e+\mathrm{Fe} x \mathrm{xv}, \\
& h \nu+\mathrm{Ni} \text { xxvI } e++\mathrm{Ni} \text { xVII. }
\end{aligned}
$$

The calculations for photoionization and electron-ion recombination for the satellite lines span several stages of computations. The target (core) wave functions are obtained from atomic structure calculations using the program SUPERSTRUCTURE [25]. These wave functions are the input for the BPRM codes and subsequent codes for electron-ion recombination. The wave function representation for the (e+ Fe xxv) system consists of a 13-level expansion for Helike Fe xxv [26], and for (e+Ni xxvII) a 17-level expansion for Ni xxvII [27] (computational details for the relativistic BPRM calculations for photoionization cross sections $\sigma_{\mathrm{PI}}$ are given in these two references). Coupled-channel calculations for $\sigma_{\mathrm{PI}}$ include both the background and the resonance structures of doubly excited autoionizing states. Radiation damping of resonances is significant for these ions and is included as described in Ref. [22]. The present computations are carried out for partial photoionization cross sections in the photoelectron energy region of the first resonance complex of the $K L L$ resonances.

Level-specific recombination cross sections $\sigma_{\mathrm{RC}}(i, s)$ are computed for the final recombined states of the electron-ion system in specified energy ranges $\left(\epsilon_{i}-\epsilon_{f}\right)$ corresponding to each satellite $s$, for all coupled symmetries $J \pi$ and final states $i$. These are summed to obtain the total $\sigma_{\mathrm{RC}}(s)$ using the program RECXS. However, the total recombination spectrum shows the satellite lines which are often overlapped by more than one line and are unresolvable for any identification. As level-specific recombination cross sections $\sigma_{\mathrm{RC}}(i, s)$ into the three bound states $1 s^{2} 2 s^{2} S_{1 / 2}, 1 s^{2} 2 p^{2} P_{1 / 2,3 / 2}^{o}$ of the recombined $(e+$ ion $)$ system correspond to the $K L L$ satellite 
lines, these cross sections are obtained from partial photoionization cross sections $\sigma_{\mathrm{PI}}(i, g)$ of the level $i$ into the ground level $g$ of the recombining ion. The satellite lines, including the overlapped ones, are now isolated in the individual level $\sigma_{\mathrm{RC}}$ and hence can be used for their identification. The specific spectroscopic transitions for the lines can, however, not be determined without prior knowledge of the energy positions for these lines. As obtained in all previous calculations, the energy positions can be determined from the existing experimental values or from an atomic structure calculations such as using SUPERSTRUCTURE.

A code SATLN is written to process the unified recombination cross sections and to obtain various quantities, such as the location and identification of the satellite lines, the dielectronic satellite strengths, $S_{\mathrm{RC}}$ and $S(s)$, and the recombination rates of the individual and integrated satellite strengths and internal checks.

\section{RESULTS AND DISCUSSION}

The dielectronic satellite lines in the $K L L$ resonant complex of He-like core ions correspond to unified BPRM $\left(e\right.$, ion) recombination cross sections $\left(\sigma_{\mathrm{RC}}\right)$ for three-electron Li-like ion with total $J \pi$ symmetries $(1 / 2)^{e},(1 / 2)^{o}$, and $(3 / 2)^{o}$. As the BPRM Hamiltonian is invariant with respect to total $J \pi$, results are obtained for each symmetry independently.

While the identification and determination of the dielectronic satellites can be done precisely, it is not straightforward to do so. Unlike the IRA methods, based partially on atomic structure calculations, the BPRM calculations do not identify resonances a priori; they form in an ab initio manner through coupling or interference of open and closed channels. Furthermore, owing to the natural overlap among resonance profiles, and uncertainty (albeit small) in the positions of the peak energies of resonances, it is necessary to examine in detail the individual $J \pi(e$,ion) recombination cross sections. Resonances corresponding to a given $J \pi$ generally separate out clearly, with some exceptions. Labels are then assigned according to the standard notation as explained in the Sec. III.

The recombination cross sections yield the absolute resonance strengths $S_{\mathrm{RC}}$ and $S(s)$ by direct integration. Although the natural overlap among resonances is quite extensive for some of the dielectronic satellites, the quantitative evaluation of most resonance strengths is not significantly affected since the dominant contribution to integrated value arises from a narrow energy range around the peak energy. The individual resonance strengths can be computed accurately except the overlapping ones with small uncertainty.

The satellite lines for iron and nickel are discussed below separately. We focus more on Fe xxv since it is of great importance in applications and detailed experimental results are available [18].

\section{A. Dielectronic satellites of Fe xxv}

All 22 satellite lines forming from the $n=2 K \alpha$ complex of $\mathrm{Fe}$ xxv have been isolated and identified in the unified

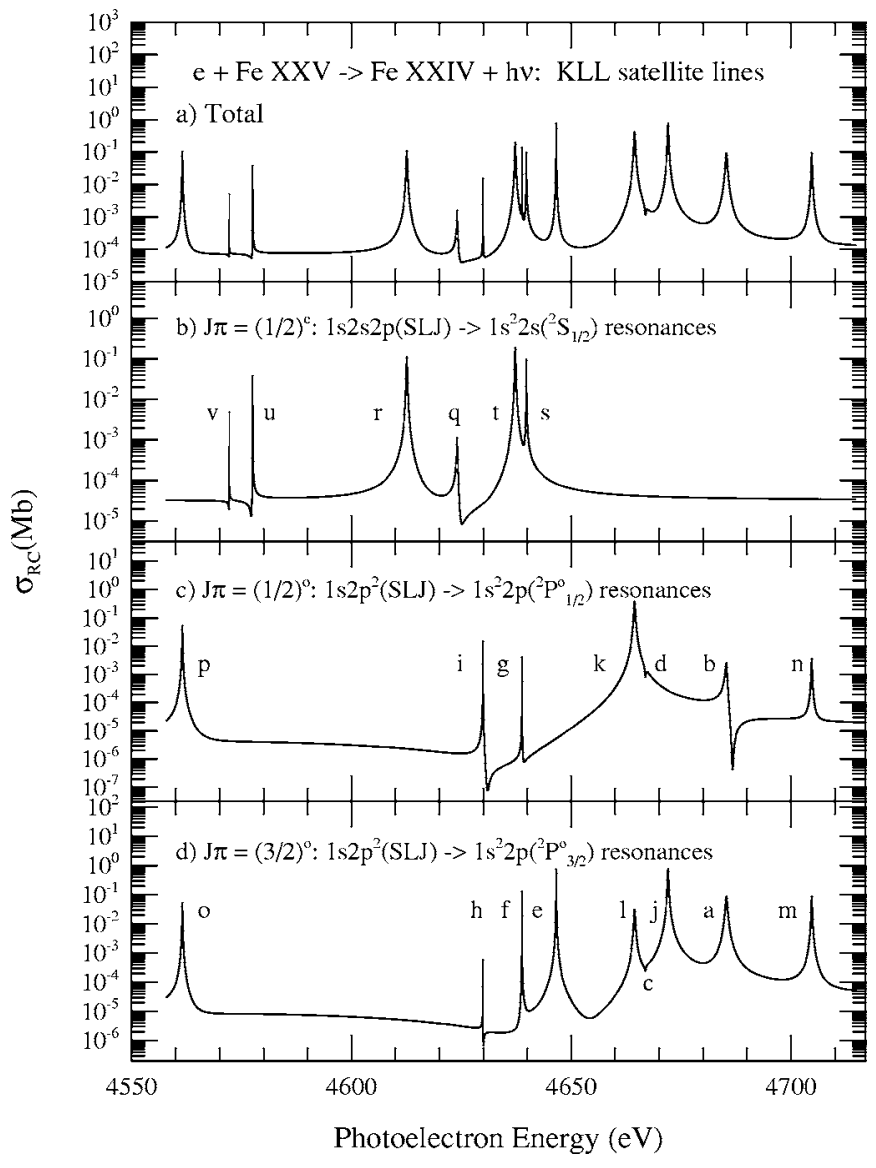

FIG. 1. Satellite lines of Fe xxv in the $K \alpha$ complex. The top panel (a) shows the total spectrum and the lower three panels show the resolved and identified lines belonging to final recombined level (b) $1 s^{2} 2 s\left({ }^{2} S_{1 / 2}\right)$, (c) $1 s^{2} 2 p\left({ }^{2} P_{1 / 2}^{o}\right)$, and (d) $1 s^{2} 2 p\left({ }^{2} P_{3 / 2}^{o}\right)$.

recombination cross sections. Figure 1 presents the computed dielectronic satellite spectra for Fe xxv in the top panel (a) and identification of individual lines corresponding to each $J \pi=(1 / 2)^{e},(1 / 2)^{o},(3 / 2)^{o}$ in panels (b)-(d), resolved at a sufficiently fine mesh to delineate complete profiles. They are labeled in the figure following the notation by Gabriel [1]. The resonances vary over orders of magnitude in cross section, with often overlapping profiles within each symmetry; thus, the intereference effects due to channel coupling are manifested in the detailed dielectronic satellites spectrum. The $J \pi$ given in Fig. 1 is the symmetry of the final autoionization channel following photoionization of the bound state with the same symmetry. However, the formation of dielectronic satellites is due to the inverse process of capture into the resonance state due to inner-shell electron impact excitation from all angular momenta. This is automatically summed over in obtaining the total unified cross sections shown in the top panel of Fig. 1. However, the background cross sections for individual $J \pi$ in the lower panels correspond to channel contribution only to that symmetry, and therefore less than the total in the top panel.

The absolute resonance strengths for Fe xxv satellite lines are obtained from integration of the recombination cross sections in Fig. 1. As the main contribution to the strengths correspond to the energy range around the peak energy, in- 
TABLE I. The $K L L$ autoionizing resonances in electron-ion recombination of $\left(e+\mathrm{Fe}_{\mathrm{xxv}}\right)$ correspond to the 22 dielectronic satellite lines. The columns represent the key notation for a satellite line, as in Ref. [1], the resonance transition, its computed energy in eV in the present work, $E(P)$, from a relativistic distorted wave calculation [11], $E(\mathrm{RDW})$, from experimental measurement, $E$ (expt.), the satellite strength in $10^{-20} \mathrm{~cm}^{2} \mathrm{eV}$ in the present work $S(P)$, and from other works $S^{a}$ [14], $S^{b}$ [15] $S^{c}$ [16], and $S^{d}$ [17] based the individual resonance approximation.

\begin{tabular}{|c|c|c|c|c|c|c|c|c|c|}
\hline Key & Transition & $E(P)$ & $E(\mathrm{RDW})$ & $E($ expt. $)$ & $S(P)$ & $S^{a}$ & $S^{b}$ & $S^{c}$ & $S^{d}$ \\
\hline$o$ & $1 s 2 s^{2}\left({ }^{2} S_{1 / 2}\right) \rightarrow 1 s^{2} 2 p\left({ }^{2} P_{3 / 2}^{o}\right)$ & 4561.5 & 4562.8 & 4553.4 & 0.89 & 0.84 & 0.91 & 0.82 & 0.94 \\
\hline$p$ & $1 s 2 s^{2}\left({ }^{2} S_{1 / 2}\right) \rightarrow 1 s^{2} 2 p\left({ }^{2} P_{1 / 2}^{o}\right)$ & 4561.5 & 4562.8 & 4553.4 & 0.88 & 0.85 & 0.92 & 0.80 & 0.91 \\
\hline$v$ & $1 s 2 p^{3} P^{o} 2 s\left({ }^{4} P_{1 / 2}^{o}\right) \rightarrow 1 s^{2} 2 s\left({ }^{2} S_{1 / 2}\right)$ & 4572.2 & 4573.9 & 4566.3 & 0.06 & 0.03 & 0.02 & 0.01 & $2.9(-3)$ \\
\hline$u$ & $1 s 2 p^{3} P^{o} 2 s\left({ }^{4} P_{3 / 2}^{o}\right) \rightarrow 1 s^{2} 2 s\left({ }^{2} S_{1 / 2}\right)$ & 4577.5 & 4578.9 & 4570.1 & 0.16 & 0.17 & 0.02 & 0.04 & 0.15 \\
\hline$r$ & $1 s 2 p{ }^{1} P^{o} 2 s\left({ }^{2} P_{1 / 2}^{o}\right) \rightarrow 1 s^{2} 2 s\left({ }^{2} S_{1 / 2}\right)$ & 4612.6 & 4615.1 & 4604.9 & 3.80 & 3.13 & 3.62 & 4.45 & 3.68 \\
\hline$q$ & $1 s 2 p{ }^{1} P^{o} 2 s\left({ }^{2} P_{3 / 2}^{o}\right) \rightarrow 1 s^{2} 2 s\left({ }^{2} S_{1 / 2}\right)$ & 4624.1 & 4625.3 & 4615.3 & 0.08 & 0.11 & 0.02 & 0.12 & $2.0(-3)$ \\
\hline$i$ & $1 s 2 p^{2}\left({ }^{4} P_{1 / 2}\right) \rightarrow 1 s^{2} 2 p\left({ }^{2} P_{1 / 2}^{o}\right)$ & 4629.9 & 4632.2 & 4624.6 & 0.08 & 0.04 & 0.02 & 0.02 & 0.03 \\
\hline$h$ & $1 s 2 p^{2}\left({ }^{4} P_{1 / 2}\right) \rightarrow 1 s^{2} 2 p\left({ }^{2} P_{3 / 2}^{o}\right)$ & 4629.9 & 4632.2 & 4624.6 & $6.0(-3)$ & $1.8(-4)$ & $2.1(-4)$ & $1.0(-4)$ & $1.0(-4)$ \\
\hline$f$ & $1 s 2 p^{2}\left({ }^{4} P_{3 / 2}\right) \rightarrow 1 s^{2} 2 p\left({ }^{2} P_{3 / 2}^{o}\right)$ & 4638.8 & 4640.5 & 4632.9 & 0.31 & 0.20 & 0.26 & 0.09 & 0.16 \\
\hline$t$ & $1 s 2 p^{3} P^{o} 2 s\left({ }^{2} P_{1 / 2}^{o}\right) \rightarrow 1 s^{2} 2 s\left({ }^{2} S_{1 / 2}\right)$ & 4637.3 & 4639.4 & 4631.2 & 5.52 & 6.35 & 5.83 & 5.28 & 5.77 \\
\hline$g$ & $1 s 2 p^{2}\left({ }^{4} P_{3 / 2}\right) \rightarrow 1 s^{2} 2 p\left({ }^{2} P_{1 / 2}^{o}\right)$ & 4638.8 & 4640.5 & 4632.9 & 0.01 & $4.5(-4)$ & $4.0(-3)$ & $4.1(-4)$ & $5.6(-4)$ \\
\hline$s$ & $1 s 2 p^{3} P^{o} 2 s\left({ }^{2} P_{3 / 2}^{o}\right) \rightarrow 1 s^{2} 2 s\left({ }^{2} S_{1 / 2}\right)$ & 4639.8 & 4642.5 & 4633.2 & 1.29 & 0.15 & 0.90 & 0.67 & 0.24 \\
\hline$e$ & $1 s 2 p^{2}\left({ }^{4} P_{5 / 2}\right) \rightarrow 1 s^{2} 2 p\left({ }^{2} P_{3 / 2}^{o}\right)$ & 4646.6 & 4648.0 & 4639.0 & 4.85 & 4.80 & 4.28 & 4.12 & 4.32 \\
\hline$k$ & $1 s 2 p^{2}\left({ }^{2} D_{3 / 2}\right) \rightarrow 1 s^{2} 2 p\left({ }^{2} P_{1 / 2}^{o}\right)$ & 4664.4 & 4664.4 & 4658.1 & 18.40 & 19.60 & 18.60 & 17.27 & 17.53 \\
\hline$d$ & $1 s 2 p^{2}\left({ }^{2} P_{1 / 2}\right) \rightarrow 1 s^{2} 2 p\left({ }^{2} P_{1 / 2}^{o}\right)$ & 4666.9 & 4668.4 & 4658.6 & 0.076 & 0.07 & 0.07 & 0.13 & 0.08 \\
\hline$l$ & $1 s 2 p^{2}\left({ }^{2} D_{3 / 2}\right) \rightarrow 1 s^{2} 2 p\left({ }^{2} P_{3 / 2}^{o}\right)$ & 4664.4 & 4666.4 & 4658.1 & 1.44 & 2.32 & 1.79 & 2.22 & 2.22 \\
\hline$c$ & $1 s 2 p^{2}\left({ }^{2} P_{1 / 2}\right) \rightarrow 1 s^{2} 2 p\left({ }^{2} P_{3 / 2}^{o}\right)$ & 4666.8 & 4668.4 & 4658.6 & 0.17 & 0.02 & 0.02 & 0.04 & 0.03 \\
\hline$j$ & $1 s 2 p^{2}\left({ }^{2} D_{5 / 2}\right) \rightarrow 1 s^{2} 2 p\left({ }^{2} P_{3 / 2}^{o}\right)$ & 4672.1 & 4673.5 & 4664.1 & 27.22 & 29.15 & 27.22 & 26.27 & 26.90 \\
\hline$b$ & $1 s 2 p^{2}\left({ }^{2} P_{3 / 2}\right) \rightarrow 1 s^{2} 2 p\left({ }^{2} P_{1 / 2}^{o}\right)$ & 4685.2 & 4686.4 & 4677.0 & 0.21 & 0.11 & 0.13 & 0.08 & 0.09 \\
\hline$a$ & $1 s 2 p^{2}\left({ }^{2} P_{3 / 2}\right) \rightarrow 1 s^{2} 2 p\left({ }^{2} P_{3 / 2}^{o}\right)$ & 4685.3 & 4686.4 & 4677.0 & 6.12 & 8.11 & 6.40 & 6.24 & 6.88 \\
\hline$m$ & $1 s 2 p^{2}\left({ }^{2} S_{1 / 2}\right) \rightarrow 1 s^{2} 2 p\left({ }^{2} P_{3 / 2}^{o}\right)$ & 4704.7 & 4706.1 & 4697.7 & 2.74 & 2.91 & 2.56 & 2.44 & 2.41 \\
\hline$n$ & $1 s 2 p^{2}\left({ }^{2} S_{1 / 2}\right) \rightarrow 1 s^{2} 2 p\left({ }^{2} P_{1 / 2}^{o}\right)$ & 4704.7 & 4706.1 & 4697.7 & 0.14 & 0.13 & 0.09 & 0.12 & 0.11 \\
\hline
\end{tabular}

terference is expected to cause very little uncertainty. However, the two exceptions are pairs $(k+d)$ and $(l+c)$, with not only overlapping profiles, but where one of the resonances in each pair is extremely weak $(<1 \%)$ and lies in the wings of the other one. A direct integration over the resonances in individual $J \pi$ cross sections would not yield the full dielectronic satellite intensity since $\sigma_{\mathrm{RC}}$ includes no contributions other than to this specific $J \pi$ symmetry. We note that the overlap and the resulting uncertainty are natural features not heretofore obtained by previous methods to our knowledge. We adopt the following procedure in calculating the dielectronic satellite intensities for resonances not clearly separable in the top panel. We obtain ratios of intensities from the lower panels and divide the total intensity accordingly. For example, the total intensity for the $(o+p)$ satellites is $1.765 \times 10^{-20} \mathrm{~cm}^{2} \mathrm{eV}$. The ratio of the $o\left[J=(3 / 2)^{\circ}\right]$ satellite to the $p\left[J=(1 / 2)^{e}\right]$ satellite is 1.006 , nearly equal, giving $S(o)=0.885$ and $S(p)=0.88$. The resulting error is insignificant since the continuum contribution is small and almost the same for both resonances.

Table I presents the computed energies and dielectronic satellites intensities for all 22 satellites. The standard notation is employed in designating the transitions. But we have arranged the dielectronic satellites in a more logical and useful energy order. It should be more useful in comparing with observations than the commonly used alphabetical order, especially where blends of dielectronic satellites are concerned on an ascending energy scale. The present energies agree well, but not exactly, with the fits to resonance profiles in Ref. [11] (done previously to compare the individual autoionization and radiative rates with the IRA calculations, and to account for radiation damping). The present energies are obviously more accurate since they correspond to peak values of resonances. The present theoretical energies agree well with previous theoretical calculations in the relativistic distorted wave approximation, usually to about $1 \mathrm{eV}$. Comparison with experimental energies can be made for the limited number of resonances resolved in the EBIT measurements [18]. A number of the experimental energies are degenerate, but are systematically lower than theoretical energies by up to $7 \mathrm{eV}(\sim 0.1 \%)$. This may be taken as the uncertainty in the present calculations.

Comparison of the present dielectronic satellite intensities with four other theoretical calculations in the IRA approximation [14-17] is also made in Table I. There is remarkable overall agreement with the previous results, given that the present method is vastly different. While this agreement is expected on physical grounds-for highly charged ions-it is gratifying nonetheless that in the limit of high $Z$ and $z$ (nuclear and ion charge, respectively) all methods appear to yield similar intensities. Whereas this is particularly true for 
TABLE II. Comparison of dielectronic resonance strengths for Fe xxv from the present unified method and previous calculations in the individual resonance approximation, with those in Table 2 of [18]. $S^{*}$ represents observed resonance strength in units of $10^{-20} \mathrm{~cm}^{2} \mathrm{eV} . R$ is the ratio of theoretical resonance strength and the measured resonance strength.

\begin{tabular}{lccccccc}
\hline \hline Key & $S^{*}$ & $R(P)$ & $R^{\mathrm{a}}$ & $R^{\mathrm{b}}$ & $R^{\mathrm{c}}$ & $R^{\mathrm{d}}$ & $R^{\mathrm{e}}$ \\
\hline$e$ & 3.63 & 1.24 & 1.02 & 1.23 & 1.10 & 1.06 & 1.11 \\
$j(+l)$ & 24.06 & 1.06 & 1.05 & 1.15 & 1.06 & 1.03 & 1.05 \\
$k(+a)$ & 21.23 & 0.91 & 0.91 & 0.98 & 0.93 & 0.86 & 0.87 \\
$m$ & 1.52 & 1.23 & 1.01 & 1.14 & 1.14 & 1.10 & 1.08 \\
$r$ & 3.42 & 0.76 & 0.77 & 0.72 & 0.72 & 0.89 & 0.73 \\
$t(+s)$ & 3.65 & 1.38 & 1.41 & 1.33 & 1.33 & 1.17 & 1.14 \\
$o$ & 0.48 & 1.25 & 1.22 & 1.29 & 1.29 & 1.16 & 1.34 \\
$p$ & 0.50 & 1.20 & 1.15 & 1.26 & 1.26 & 1.10 & 1.25 \\
\hline \hline
\end{tabular}

${ }^{\mathrm{a}}$ Reference [11].

${ }^{\mathrm{b}}$ Reference [14].

${ }^{\mathrm{c}}$ Reference [15].

${ }^{\mathrm{d}}$ Reference [16].

${ }^{\mathrm{e}}$ Reference [17].

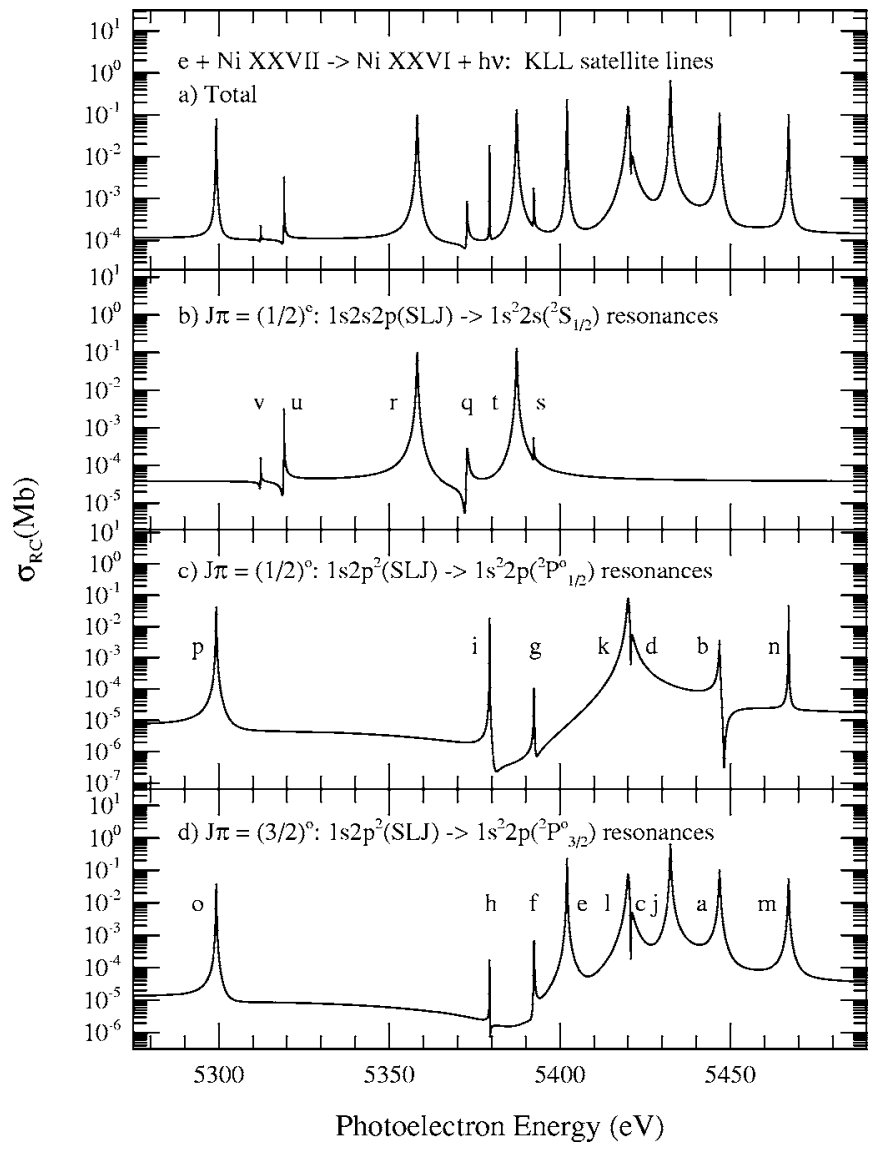

FIG. 2. Satellite lines of Ni XxvII in the $K \alpha$ complex. The top panel (a) shows the total spectrum and the lower three panels show the resolved and identified lines belonging to final recombined levels (b) $1 s^{2} 2 s\left({ }^{2} S_{1 / 2}\right)$, (c) $1 s^{2} 2 p\left({ }^{2} P_{1 / 2}^{o}\right)$, and (d) $1 s^{2} 2 p\left({ }^{2} P_{3 / 2}^{o}\right)$.
TABLE III. The computed energies (eV) and strengths of dielectronic satellite lines in the $K L L$ resonance complex in electron recombination spectrum of $(e+\mathrm{Ni}$ XXVII $)$ from the unified method.

\begin{tabular}{lccc}
\hline \hline Key & Transition & $E(P)$ & $S(P)$ \\
\hline$o$ & $1 s 2 s^{2}\left({ }^{2} S_{1 / 2}\right) \rightarrow 1 s^{2} 2 p\left({ }^{2} P_{3 / 2}^{o}\right)$ & 5296.0 & 0.99 \\
$p$ & $1 s 2 s^{2}\left({ }^{2} S_{1 / 2}\right) \rightarrow 1 s^{2} 2 p\left({ }^{2} P^{o}\right)$ & 5296.0 & 1.11 \\
$v$ & $1 s 2 p{ }^{3} P^{o} 2 s\left({ }^{4} P_{1 / 2}^{o}\right) \rightarrow 1 s^{2} 2 s\left({ }^{2} S_{1 / 2}\right)$ & 5312.3 & 0.07 \\
$u$ & $1 s 2 p{ }^{3} P^{o} 2 s\left({ }^{4} P_{3 / 2}^{o}\right) \rightarrow 1 s^{2} 2 s\left({ }^{2} S_{1 / 2}\right)$ & 5321.2 & 0.18 \\
$r$ & $1 s 2 p{ }^{1} P^{o} 2 s\left({ }^{2} P_{1 / 2}^{o}\right) \rightarrow 1 s^{2} 2 s\left({ }^{2} S_{1 / 2}\right)$ & 5357.2 & 4.26 \\
$q$ & $1 s 2 p{ }^{1} P^{o} 2 s\left({ }^{2} P_{3 / 2}^{o}\right) \rightarrow 1 s^{2} 2 s\left({ }^{2} S_{1 / 2}\right)$ & 5372.2 & 0.11 \\
$i$ & $1 s 2 p^{2}\left({ }^{4} P_{1 / 2}\right) \rightarrow 1 s^{2} 2 p\left({ }^{2} P_{1 / 2}^{o}\right)$ & 5379.4 & 0.28 \\
$h$ & $1 s 2 p^{2}\left({ }^{4} P_{1 / 2}\right) \rightarrow 1 s^{2} 2 p\left({ }^{2} P_{3 / 2}^{o}\right)$ & 5379.4 & $4.3(-3)$ \\
$t$ & $1 s 2 p{ }^{3} P^{o} 2 s\left({ }^{2} P_{1 / 2}^{o}\right) \rightarrow 1 s^{2} 2 s\left({ }^{2} S_{1 / 2}\right)$ & 5385.8 & 5.36 \\
$f$ & $1 s 2 p^{2}\left({ }^{4} P_{3 / 2}\right) \rightarrow 1 s^{2} 2 p\left({ }^{2} P_{3 / 2}^{o}\right)$ & 5393.3 & 0.03 \\
$g$ & $1 s 2 p^{2}\left({ }^{4} P_{3 / 2}\right) \rightarrow 1 s^{2} 2 p\left({ }^{2} P_{1 / 2}^{o}\right)$ & 5394.3 & 0.04 \\
$s$ & $1 s 2 p{ }^{3} P^{o} 2 s\left({ }^{2} P_{3 / 2}^{o}\right) \rightarrow 1 s^{2} 2 s\left({ }^{2} S_{1 / 2}\right)$ & 5394.3 & 0.13 \\
$e$ & $1 s 2 p^{2}\left({ }^{4} P_{5 / 2}\right) \rightarrow 1 s^{2} 2 p\left({ }^{2} P_{3 / 2}^{o}\right)$ & 5402.1 & 5.40 \\
$k$ & $1 s 2 p^{2}\left({ }^{2} D_{3 / 2}\right) \rightarrow 1 s^{2} 2 p\left({ }^{2} P_{1 / 2}^{o}\right)$ & 5414.4 & 6.38 \\
$l$ & $1 s 2 p^{2}\left({ }^{2} D_{3 / 2}\right) \rightarrow 1 s^{2} 2 p\left({ }^{2} P_{3 / 2}^{o}\right)$ & 5414.4 & 6.10 \\
$d$ & $1 s 2 p^{2}\left({ }^{2} P_{1 / 2}\right) \rightarrow 1 s^{2} 2 p\left({ }^{2} P_{1 / 2}^{o}\right)$ & 5424.1 & 1.16 \\
$c$ & $1 s 2 p^{2}\left({ }^{2} P_{1 / 2}\right) \rightarrow 1 s^{2} 2 p\left({ }^{2} P_{3 / 2}^{o}\right)$ & 5424.1 & 1.15 \\
$j$ & $1 s 2 p^{2}\left({ }^{2} D_{5 / 2}\right) \rightarrow 1 s^{2} 2 p\left({ }^{2} P_{3 / 2}^{o}\right)$ & 5427.6 & 25.0 \\
$b$ & $1 s 2 p^{2}\left({ }^{2} P_{3 / 2}\right) \rightarrow 1 s^{2} 2 p\left({ }^{2} P_{1 / 2}^{o}\right)$ & 5447.7 & 0.23 \\
$a$ & $1 s 2 p^{2}\left({ }^{2} P_{3 / 2}\right) \rightarrow 1 s^{2} 2 p\left({ }^{2} P_{3 / 2}^{o}\right)$ & 5447.7 & 5.80 \\
\hline \hline & $1 s 2 p^{2}\left({ }^{2} S_{1 / 2}\right) \rightarrow 1 s^{2} 2 p\left({ }^{2} P_{3 / 2}^{o}\right)$ & 5468.1 & 2.33 \\
& $1 s 2 p^{2}\left({ }^{2} S_{1 / 2}\right) \rightarrow 1 s^{2} 2 p\left({ }^{2} P_{1 / 2}^{o}\right)$ & 5468.1 & 0.66 \\
\hline & & & \\
& & &
\end{tabular}

the stronger satellites, such as $a, j, k, l, r$, and $t$ [all with $\left.S(s)>10^{-20} \mathrm{~cm}^{2} \mathrm{eV}\right]$ there are considerable differences for the weakest ones, such as $c$ and $d$. At least a part of these differences may be attributed to configuration interaction and overlapping profiles, and hence intereference effects which preferentially affect the relatively weaker satellite intensities. More highly resolved measurements should be able to verify the actual extent of these differences.

The present results, with blended satellites as observed in the EBIT experiment [18], are compared with the experiment and other work in Table II. The comparison is not quite direct, and involves corrections for polarization in the experimental values as explained in Ref. [18]. We compute the ratio $R=G(s / w) S(s) / S^{*}$ using the polarization correction factors $G(s / w)$ provided in [18] for each satellite. $S^{*}$ represents the observed resonance strength in units of $10^{-20} \mathrm{~cm}^{2} \mathrm{eV}$. The agreement for the ratio $R$ of theoretical to experimental values is better than $25 \%$, except for the blend $(t+s)$ where it is $38 \%$. These figures may be taken to be the combined theoretical and experimental uncertainties in the dielectronic satellite intensities. However, there are some small but possibly significant differences. For example, the theoretical $S(o)$ is slightly greater than $S(p)$; the experimental values are the reverse. The largest differences with the IRA calculations are for dielectronic satellite intensities most susceptible to interference effects. These are the resonances corresponding to $d$ and $c$ satellites that are seen to lie on the wings of the 
much stronger satellites $k$ and $l$, respectively. In principle, therefore, individual resonance strengths cannot be determined accurately for the weaker satellites owing to the strong interaction with the stronger competing channels. While $S(c)$ and $S(d)$ are very small, we have attempted to obtain these from integration in a small energy range around the sharp dip seen in both cases. The present values are much larger than previous IRA calculations that do not take into account interference effects. The differences between the present and the previous calculations may be taken to be the maximum uncertainty for the strengths of the $c$ and $d$ satellites.

\section{B. Dielectronic satellites of Ni xxvII}

The $22 n=2$ satellite lines of Ni xxvII are resolvable, as expected for this highly charged ion, in the unified recombination cross sections. The lines can be easily identified from individual level spectra as shown in Fig. 2. The basic features of the satellite lines are similar to those for Fe xxv. With higher charge, the blended lines $d$ and $c, l$ of $\mathrm{Ni}$ XXVII are better resolved than Fe xxv. However, variations in the resonance strengths of individual lines are noted between these two ions. Table III presents the computed energies and strengths of the satellite lines of $K L L$ resonances in the unified $\sigma_{\mathrm{RC}}$.

\section{CONCLUSION}

The main conclusions of this work are as follows.

(1) In addition to providing a theoretical and computational framework for self-consistent and unified treatment of photoionization and radiative and dielectronic recombination, the unified method is now applied to accurate calculation of dielectronic satellite intensities. The method is different from previous theoretical methods based on the individual resonance approximation. The approach utilizes the powerful $R$-matrix method in contrast to the distorted wave method used in many previous calculations.

(2) As expected for very highly charged ions, the present unified dielectronic resonance strengths are in good agreement with those computed in previous works using the individual resonance approximation. Nevertheless, the results demonstrate that intereference effects, due to channel coupling and configuration interaction included in the unified method, could be significant for more complex ions.

(3) The present dielectronic resonance strengths agree well with experimental measurements, as resolved. However, in the absence of experimental data for each individual satellite it is difficult to ascertain the precise level of agreement, except that is generally within $25 \%$.

(4) Dielectronic satellite intensities may be computed using the satellite strengths tabulated herein. However, explicit calculation of satellite to resonance intensity line ratios $I(s) / I(w)$ are in progress. That would facilitate applications to X-ray spectroscopy of laboratory and astrophysical plasmas across a wide range of temperatures, as well as further comparisons with previous theoretical results.

\section{ACKNOWLEDGMENTS}

This work was partially supported by the NASA Astrophysical Theory Program. The computational work was carried out on the Cray SV1 and the Cray X1 at the Ohio Supercomputer Center in Columbus Ohio.
[1] A. H. Gabriel, Mon. Not. R. Astron. Soc. 160, 99 (1972).

[2] C. P. Bhalla, A. H. Gabriel, and L. P. Presnyakov, Mon. Not. R. Astron. Soc. 172, 359 (1975).

[3] M. Arnaud and J. Raymond, Astrophys. J. 398, 394 (1992).

[4] J. Oelgoetz and A. K. Pradhan, Mon. Not. R. Astron. Soc. 327, L42 (2001).

[5] J. Oelgoetz and A. K. Pradhan, Mon. Not. R. Astron. Soc. 354, 1093 (2004).

[6] Y-D. Xu, R. Narayan, E. Quataert, F. Yuan, and F. K. Baganoff, Astrophys. J. 640, 319 (2006).

[7] S. N. Nahar and A. K. Pradhan, Phys. Rev. Lett. 68, 1488 (1992).

[8] S. N. Nahar and A. K. Pradhan, Phys. Rev. A 49, 1816 (1994).

[9] S. N. Nahar and A. K. Pradhan, Radiat. Phys. Chem. 70, 323 (2004).

[10] S. N. Nahar and A. K. Pradhan, Astrophys. J., Suppl. Ser. 162, 417 (2006).

[11] A. K. Pradhan and H. L. Zhang, J. Phys. B 30, L571 (1997).

[12] A. K. Pradhan, Astrophys. J. Lett. 545, L165 (2000).

[13] S. N. Nahar, A. K. Pradhan, and H. L. Zhang, Phys. Rev. A 63, 060701(R) (2001).

[14] L. A. Vainshtein and U. I. Safronova, At. Data Nucl. Data Tables 25, 49 (1978).
[15] F. Bely-Dubau, J. Dubau, P. Faucher, and A. H. Gabriel, Mon. Not. R. Astron. Soc. 198, 239 (1982).

[16] M. H. Chen, At. Data Nucl. Data Tables 34, 301 (1986).

[17] J. Nilsen, At. Data Nucl. Data Tables 38, 339 (1988).

[18] P. Beiersdorfer, T. W. Phillips, K. L. Wong, R. E. Marrs, and D. A. Vogel, Phys. Rev. A 46, 3812 (1992).

[19] D. G. Hummer, K. A. Berrington, W. Eissner, A. K. Pradhan, H. E. Saraph, and J. A. Tully, Astron. Astrophys. 279, 298 (1993).

[20] S. N. Nahar, A. K. Pradhan, and H. L. Zhang, Astrophys. J., Suppl. Ser. 131, 375 (2000).

[21] K. Sakimoto, M. Terao, and K. A. Berrington, Phys. Rev. A 42, 291 (1990).

[22] H. L. Zhang, S. N. Nahar, and A. K. Pradhan, J. Phys. B 32, 1459 (1999).

[23] S. N. Nahar, Phys. Rev. A 53, 2417 (1996).

[24] R. H. Bell and M. J. Seaton, J. Phys. B 18, 1589 (1985).

[25] W. Eissner, M. Jones, and N. Nussbaumer, Comput. Phys. Commun. 8, 270 (1974).

[26] S. N. Nahar, A. K. Pradhan, and H. L. Zhang, Astrophys. J., Suppl. Ser. 133, 255 (2001).

[27] S. N. Nahar, Astrophys. J., Suppl. Ser. 158, 80 (2005). 